\title{
Ética y aprendizaje en cirugía laparoscópica
}

\author{
Jara Rascón J, Subirá Ríos D. \\ Hospital General Universitario Gregorio Marañón. Madrid. \\ Actas Urol Esp 2006; 30 (5): 474-478

\section{RESUMEN} \\ ÉTICA Y APRENDIZAJE EN CIRUGÍA LAPAROSCÓPICA
}

La adquisición de habilidades quirúrgicas es un asunto de la mayor importancia durante el periodo de aprendizaje que supone la realización de la especialidad de Urología. Por ello, la progresiva implantación de la laparoscopia en nuestra actividad asistencial debe ir seguida de un plan de aprendizaje escalonado, como respuesta a esta nueva necesidad de formación, que debería ser cuidadosamente diseñado para no causar perjuicio alguno, en ningún caso, a los pacientes intervenidos con esta nueva modalidad de abordaje quirúrgico.

Partiendo de la aceptación de esta premisa, se debe enfocar cada caso y cada indicación en particular, asegurando que se preservan los principios básicos de la ética médica (beneficencia, no maleficencia, justicia y autonomía). Para ello, habrá que sopesar los riesgos probables conforme a la propia experiencia o directamente relacionados con el tipo de intervención. Con respecto a esto, en el presente artículo se esbozan las diferentes opciones para llevar a cabo un plan éticamente aceptable de formación en laparoscopia urológica, tomando como punto de referencia la experiencia en prostatectomía laparoscópica. Asimismo, se exponen las razones justificativas de la denominada "reconversión programada" y, finalmente, se plantea la discusión respecto a qué se debe informar y qué cantidad de información se debe dar para que los pacientes puedan ejercer su capacidad de decisión de modo real.

Palabras clave: Ética. Laparoscopia. Prostatectomía.

\section{ABSTRACT \\ ETHICS AND TRAINING IN LAPAROSCOPIC SURGERY}

Teaching operative skills are of paramount importance to urology training, specially in the period of time of the residency. Because of that, the introduction of laparoscopy in our surgical activity must be followed by a careful planning of progressive training as an answer to this new need that would be well designed trying not to damage in whatever possible way to the patients operated with this new modality of surgical intervention.

In this sense, each case and each indication of laparoscopy must be clarified individually, taking into account the basic principles of medical ethics (beneficency, no maleficency, justice and autonomy). So, it will be neccesary to decide about possible risks due to the experience of the surgeon or due to the modality of surgery. In this article, different options of training plannings in laparoscopy are mentioned taking as a reference the experience in laparoscopic prostatectomy, reasons of "prepared reconversion" are exposed and, finally, the authors explain the discussion about the subject and the extent of information offered to the patients looking for the best possibilities of decision making by the patients. 
D esde que la laparoscopia fue introducida en la práctica quirúrgica urológica, se ha producido un incremento progresivo del número de sus posibles aplicaciones en nuestro ámbito de trabajo, incluyéndose como tales tanto complejas cirugías de exéresis oncológica como cuidadosas técnicas reconstructivas. Esta situación está trayendo nuevos y prometedores vientos de cambio a nuestra especialidad pero estos procedimientos exigen para su correcta realización un previo entrenamiento para adquirir la suficiente destreza manual, con el fin de no provocar ningún daño evitable a los pacientes que van a ser sometidos a estas técnicas.

Este modelo de actuación que, en realidad, es inherente a toda la práctica quirúrgica adquiere unas connotaciones éticas especiales al referirnos a la cirugía laparoscópica. En primer lugar, para las patologías operables mediante laparoscopia ya se dispone de otra alternativa quirúrgica: la cirugía abierta convencional. Por tanto, la indicación de cirugía laparoscópica sólo tendrá sentido para cada paciente concreto si se espera conseguir un mayor beneficio (menor morbilidad potencial) también en la etapa de aprendizaje, con este tipo de cirugía frente a la cirugía abierta. En segundo lugar, la existencia de una curva de aprendizaje "escarpada", asumida como tal, por la práctica totalidad de los cirujanos expertos en laparoscopia ${ }^{1}$, deberá ser planificada de modo que no dé lugar a situaciones limitrofes con la experimentación en humanos en una situación que no es la de un ensayo clínico controlado. Como tercera consideración, se debe tener en cuenta el derecho del paciente a una información veraz sobre el proceso quirúrgico a que va a ser sometido, respetando su libertad a elegir lo que él considere como "la mejor opción", ya que estamos hablando de diferentes modos de abordaje quirúrgico para las mismas indicaciones. Por último, en la asistencia sanitaria pública, habrá que considerar adicionalmente la competitividad en la ecuación coste-beneficio de estas nuevas técnicas, tanto a nivel económico como social, para justificar su introducción como práctica habitual, sin dar lugar a demoras en las listas de espera del resto de los pacientes que, en una teórica consideración a priori, podrían resultar perjudicados debido al aumento del número de horas de ocupación de quirófanos ocasionado por la fase de aprendizaje de la cirugía laparoscópica.

Aunque este planteamiento global de la situación pueda parecer demasiado exigente, no es en absoluto utópico si se considera de modo pormenorizado, tal como se expone a continuación, y su aceptación debería marcar el estándar mínimo para una instauración de la cirugía laparoscópica compatible con los criterios de buena práctica clínica. Se evitarán así situaciones traumáticas innecesarias, falsamente autojustificadas por la obligada curva de aprendizaje individual.

En concreto, el aprendizaje en laparoscopia puede ser realizado mediante diversas modalidades tales como el pelvitrainer, la realización de laparoscopia con animales de experimentación o en cadáveres $\mathrm{y}$, en centros especialmente dotados, mediante simuladores de realidad virtual. Sea cual fuere la modalidad elegida, se deberá asumir un alto coste tanto en tiempo de dedicación como en recursos materiales. Posiblemente la mejor opción, no siempre fácil de planificar, sea el copilotaje con un cirujano laparoscopista ya experimentado pero ¿por cuánto tiempo?. Evidentemente la necesidad de formación específica, debidamente interiorizada antes de iniciar una cirugía de este tipo en humanos, hace muy conveniente un periodo de entrenamiento básico con animales de experimentación, utilizándose animales porcinos preferentemente en la mayoría de los centros en que esta opción es posible. Mediante esta fase, es posible familiarizarse con las vías de abordaje y la colocación de los trócares, iniciándose el logro de habilidades necesarias para realizar la disección endoscópica y la sutura de tejidos. En este tipo de entrenamiento se ha propuesto la nefrectomía como una buena intervención para comenzar ${ }^{2}$. Así, parece esperable que cuanta mayor soltura o destreza se haya adquirido mediante la cirugía experimental, sea menor el tiempo necesario para transferir dicha destreza a la práctica quirúrgica en humanos, con la asistencia técnica posterior de cirujanos experimentados en laparoscopia.

Con estas premisas de actuación, se intenta preservar el principio de beneficencia, que es el principio ético que obliga no sólo a no hacer el mal, sino a hacer el bien, según afirma Gracia ${ }^{3}$. Es decir, se estaría capacitado para realizar la 
cirugía laparoscópica sólo si se piensa que se es capaz de aportar un beneficio con estas técnicas a cada paciente concreto. Si no es esa aún la situación, debería asumirse, aunque cueste, que se debe aún mantener la fase de entrenamiento.

Pasando a la siguiente consideración sobre la curva de aprendizaje, como ya se ha mencionado, ésta no puede erigirse en argumento justificativo de una mayor morbilidad (supuestamente inevitable) sobre los primeros pacientes que se van a someter a estas técnicas en un centro determinado que decida iniciar su andadura en este recorrido quirúrgico. De hecho, la posibilidad de limitar el tiempo laparoscópico de esta cirugía, finalizándola como cirugía abierta al llegar a un número de horas predeterminado, puede evitar riesgos innecesarios de sobreexposición anestésica. Esta decisión se ha definido como "reconversión programada" 4 , contrariamente a los que consideran este tipo de proceder como un fracaso del acto quirúrgico, esta actuación debería ser adoptada como una de las condiciones previas exigibles para realizar la curva de aprendizaje sin riesgos. En casos en los que la ecuación riesgo/beneficio sea dudosa por su duración, se debería consultar al Comité de Ética Asistencial de cada centro, debiendo informar de las alternativas terapéuticas existentes. De este modo, asumiendo una posible reconversión, con un aumento del tiempo quirúrgico preestablecido como aceptable y sin añadir costes ni morbilidad, se puede lograr el aprendizaje progresivo de técnicas tales como la prostatectomía radical. Optar por realizar la técnica por vía laparoscópica hasta el final, independientemente de su duración, no parece asumible desde el punto de vista ético si se afirma estar buscando el beneficio del paciente.

Con respecto a esto, se debe recordar que la regla clásica de la ética médica primum non nocere (primero no hacer daño) viene definida en buena medida por la lex artis y los criterios de indicación, de no indicación y de contraindicación. Esto permite entender que el contenido de este principio haya que definirlo en cada momento, reconsiderando en cada caso la ecuación riesgo/beneficio potencial para cada paciente, según el desarrollo de la habilidad del cirujano. Así, el antecedente de cirugía previa o la extensión del proceso pueden contribuir a una mayor dificul- tad de la técnica ante una misma indicación haciendo no aconsejable la intervención mediante laparoscopia en estos casos.

Otro aspecto que merece especial atención es el de la información que debemos dar a nuestros pacientes en esta fase de primeras cirugías laparoscópicas sin experiencia aún valorable. Por una parte, es obvio que se debe disponer de un consentimiento informado específico en el que, de acuerdo con lo establecido en la Ley 41/2002 básica reguladora de la autonomía del paciente $y$ de derechos y obligaciones en materia de información y documentación clinica, se informe al paciente de las consecuencias relevantes o de importancia esperables de la intervención, los riesgos relacionados con las circunstancias personales o profesionales del paciente, los riesgos probables conforme a la experiencia o directamente relacionados con el tipo de intervención (Artic. 10). Por otra parte, esta exigencia de exhaustividad ha dado lugar a diferentes opiniones respecto a su idoneidad y posibilidades de aplicación. Autores como Herranz ${ }^{5}$ opinan que es preferible que sobren y no que falten, apoyándose en que la rutina se encargará de desgastar las aristas de esas normas y considerando la doctrina básica del Código de Nüremberg válida y aplicable en su intensidad original.

Para otros autores ${ }^{6}$ apartarse de la fórmula estándar del consentimiento informado podría suponer además un retorno al paternalismo médico, en el que éste, basándose en argumentos culturales, podría ocultar o manipular la información a fin de asegurarse de que las decisiones sean acordes con su propio juicio clínico.

Pensamos que las normas éticas o los derechos humanos son universales, no porque sean reconocidos por ciertos gobiernos o culturas, sino porque es universal la dignidad humana, sobre la que están fundamentados. El respeto a esta dignidad humana es lo que obliga a los profesionales de la asistencia sanitaria a obtener el consentimiento de los pacientes de manera que sea comprensible y acorde con el lenguaje, las costumbres y la cultura de la persona. Informar de este modo respecto a las ventajas de la laparoscopia y, a la vez, de sus limitaciones y de la posibilidad de reconversión de la técnica, puede resultar para algunos dificultoso, pero en ninguna ocasión 
puede ser considerado como válido el engaño o la información incompleta como método para obtener la aceptación del paciente para someterse al proceso quirúrgico. Por eso, la única alternativa aceptable será mejorar nuestra capacidad de comunicación para dar una información convincente y veraz sobre las ventajas de este nuevo tipo de cirugía, sin soslayar los riesgos, de modo en parte similar a las explicaciones que se facilitan para obtener el consentimiento en los ensayos de investigación clínica.

Finalmente, al establecer un programa de cirugía laparoscópica, con asignación de días concretos para su realización y la consiguiente ocupación de quirófanos para que esta práctica quirúrgica no sea anecdótica, se debe vigilar que se mantengan los criterios de justicia que deben regir nuestra toma de decisiones respecto a los pacientes, controlando la equidad en las listas de espera para no perjudicar a unos (los que rehúsen someterse a esta técnica) demorando su prioridad quirúrgica, ni privilegiar a otros (los que la acepten) concediéndoles dicha prioridad.

Se ha especulado mucho a este respecto ya que, en un Sistema de Salud público, con una limitación clara de los recursos asistenciales y listas de espera quirúrgica en ocasiones no fácilmente controlables, puede a veces ser difícil establecer criterios de absoluta justicia en la decisión de a quién operar en primer lugar. Por ello, cuando la limitación de recursos disponibles así lo justifique, se deberá priorizar la asistencia valorando la adecuación y eficacia en el tiempo de las técnicas utilizadas. De hecho, el propio Tribunal Constitucional se ha hecho eco de estas consideraciones al analizar diversas prestaciones del Sistema de Seguridad Social, admitiendo que su idoneidad queda condicionada por los medios disponibles, valorando la posibilidad de modificar sus condiciones en función de las situaciones de necesidad a satisfacer. La protección del derecho y la equidad del sistema no serán efectivas si no se garantiza un nivel adecuado y mínimo de calidad en las prestaciones, condicionado por una situación de recursos económicos insuficientes, en la que los poderes públicos están obligados a conseguir la mejor relación calidad-coste con el fin de hacer llegar sus servicios a la mayor parte de la población.
Una manera de evitar estos potenciales conflictos en las listas de espera pasaría también por admitir el principio de posible "reconversión programada" de la cirugía laparoscópica para limitar los tiempos quirúrgicos y hacer esta técnica así más competitiva, sobre todo en las primeras fases de su introducción en la práctica quirúrgica. Otra modalidad posible es la asignación, por parte de la dirección de los centros, de horas "extra" de quirófano, para no interferir con la cirugía habitualmente programada. De todos modos, sea cual sea la decisión propiciada, la aspiración del proceso de establecimiento de prioridades debería ser alcanzar una situación en la que las decisiones adoptadas sean razonables y públicamente defendibles, ya que si trascendieran a la opinión pública, podrían ser consideradas como aceptables y justas. Estas preocupación por la equidad ha llevado a que en algunos países europeos, como Dinamarca, las autoridades sanitarias hayan pedido a sus organismos competentes, a través del Danish Council of Ethics, la elaboración de recomendaciones para asegurar que el proceso de establecimiento de prioridades se llevara a cabo de un modo transparente y que tuviera en cuenta los intereses de todos los agentes involucrados.

Respecto a la discusión sobre los costes totales que estas técnicas pueden suponer en un Sistema de Salud Público, resultan clarificadores los estudios que ya se han realizado centrados sobre aspectos económicos. Así, al comparar en términos económicos la nefrectomía radical y la nefroureterectomía laparoscópicas frente a las correspondientes técnicas abiertas ${ }^{7}$, se observa que los costes de la laparoscopia, inicialmente más altos durante la curva de aprendizaje, se transforman en los más rentables ( $12 \%$ y $6 \%$ más bajos que los costes de la cirugía abierta respectivamente para cada una de las técnicas comparadas) al aumentar la experiencia de los cirujanos, obteniéndose un menor tiempo de ocupación de quirófanos, una estancia hospitalaria más corta y una recuperación del paciente más rápida.

Teniendo en cuenta todo lo anteriormente mencionado, se puede deducir que un correcto aprendizaje en laparoscopia no requiere únicamente conocer los límites éticos de nuestra actuación profesional, que están bien delimitados en general, sino avanzar en la ética del propio pro- 
fesional. Solamente unos profesionales que tengan un criterio ético bien definido, podrán establecer los criterios concretos que dirijan su trabajo diario, limitándolo en un sentido para potenciarlo en otro. La legislación o los códigos deontológicos, aún establecidos con la celeridad a la que los adelantos tecnológicos nos impulsan, no podrán encauzar adecuadamente nuestro trabajo: deberemos hacerlo los propios médicos. Si se actúa de esta manera, se entenderá fácilmente que la ética no es un factor limitante de la investigación o de la actuación profesional, sino una decidida apuesta por el ser humano y su dignidad.

\section{REFERENCIAS}

1. Poulakis V, Dillenburg W, Moeckel M, de Vries R, Witzsch U, Zumbe J. Laparoscopic radical prostatectomy: prospective evaluation of the learning curve. Eur Urol. 2005;47(2): 167175.
2. Novas S, Ruibal M, González M. Docencia en urología laparoscópica: Problemas. Soluciones. Actas Urol Esp. 2005;29 (3):241-243.

3. Gracia D. La deliberación moral: el método de la ética clínica. Med Clin. 2001;117(1):18-23.

4. Llorente C, Carrera C, Sánchez M, de la Morena JM, González F, Martínez J, Rengifo D. Implantación de un programa de prostatectomía radical laparoscópica. Actas Urol Esp. 2005;29 (4):349-354.

5. Herranz G. La bioética en la investigación del ser humano. Medicina y ética 1994;3:315-333.

6. Simón P, Júdez J. Consentimiento informado. Med Clin (Barc) 2001;117:99-106.

7. Meraney AM, Gill IS. Financial analysis of open versus laparoscopic radical nephrectomy and nephroureterectomy. J Urol 2002;167:1757-1762.

Dr. J. Jara Rascón

E-mail: JJR00002@terra.es 